\title{
The Emergence of "Syn-Epistemic Wholeness" from Dialectic Synergy of Disciplines: A Transdisciplinary Social Pedagogic Model
}

\author{
Iro Mylonakou-Keke \\ Pedagogical Faculty of Primary Education, School of Education, National and Kapodistrian University of Athens, \\ Athens, Greece \\ Email: imylon@primedu.uoa.gr
}

Received 28 September 2015; accepted 24 October 2015; published 27 October 2015

Copyright (C) 2015 by author and Scientific Research Publishing Inc. This work is licensed under the Creative Commons Attribution International License (CC BY). http://creativecommons.org/licenses/by/4.0/

(c) (i) Open Access

\section{Abstract}

Transdisciplinarity offers a different philosophy and approach both of how to effectively address issues/problems and how to improve and change difficult situations we have been experiencing in the modern world, in different and multiple levels of reality and under conditions of complexity and uncertainty. Addressing these problems becomes precisely effective because of the way transdisciplinarity achieves dynamic collaboration and integration among disciplines, epistemologies and methodologies. We could argue that, in essence, an ongoing "dialectic synergy" between disciplines, methodologies, scientists and researchers in various forms and at many interactive levels is created. The constant dialectic synergies generate the "Syn-epistemic Wholeness". Synepistemic wholeness is a new "entity" that transcends the involved disciplines (or sciences) without replacing them; it rather enriches them by offering the potential of a synchronous, harmonic function in a flexible and constantly evolving methodological framework. By this logic, the Syn-epistemic Wholeness allows the emergence of a new general perception, the essence of a new "paradigm", which has new philosophy, theory, epistemology as well as new research strategies and practices and which offers rich possibilities and alternatives to address complex, multiparameter and multifarious problems. "Syn-epistemic Wholeness" would be an alternative term for Transdisciplinarity. This paper presents a Transdisciplinary Social Pedagogic Model, which emerged through extensive research projects and carried out within school communities in Greece. The research projects have the general orientation to address and prevent pupils' antisocial behaviors. The Model motivates a lot of transdisciplinary dialectic synergies that incrementally and improvingly developed among: (a) the involved disciplines, epistemologies and methodologies and (b) among scientists, researchers and all other stakeholders, who were those who directly or indirectly were involved in the school and in the wider community. 


\title{
Keywords
}

\author{
Syn-Epistemic Wholeness, Transdisciplinary Social Pedagogic Model, Dialectic Synergy, School \\ Community
}

\section{Introduction}

Modern societies have been facing a large number of an ever increasing complexity of problems that the scientific community is called for encountering effectively. At the same time, it is often observed an intense fragmentation of the unity of scientific knowledge. According to Ilya Prigogine (the winner of the Nobel Prize, in Chemistry), this fragmentation has come from our ability to analyze the problems in the simplest possible parts of which they are composed. As he claims, in the modern western culture we do this so well that most of the time we forget to put these parts back in place (Prigogine \& Stengers, 1984).

The fragmented knowledge facilitates research and sometimes teaching, but it remains stubbornly "docked" in analytical approaches with all epistemological and methodological problems that these approaches entail. Additionally, in some cases the communication between scientists, even when they approach the same problem, becomes difficult or impossible. These scientists seem as if "they live in parallel cognitive universes" (Kekes, 2007: p. 20), and they are forced to often use methodological and linguistic codes that are incompatible between them.

Interdisciplinarity and Transdisciplinarity (at a higher level) have managed to take scientists out of the isolation of the "universe of their discipline", break and transgress the boundaries, make them collaborate, integrate different knowledge, get synchronized in a combined function and eventually to become more effective in dealing with mainly complex issues.

In the limited context of this paper we will expand our discussion about neither interdisciplinarity nor its comparison with transdisciplinarity. Moreover, many scholars have extensively referred to it (Klein, 2010; Leavy, 2011; Nicolescu, 1997; Wickson et al., 2006). We will focus only on the integration that is achieved when there is collaboration between disciplines, that is: (a) when there is interdisciplinarity, there is "collaboration with varying levels of integration of concepts, theories, methods and findings"; whereas (b) when there is transdisciplinarity, there is "collaboration with high levels of integration causing the development of new conceptual, theoretical and methodological frame works” (Leavy, 2011: p. 33).

As far as transdisciplinarity is concerned, there have been studies made by important thinkers, especially in recent years (Gibbs, 2015; Hirsch Hadorn et al., 2008; Klein, 2008; Leavy, 2011; Nicolescu, 2002), thinkers who come from various disciplines. These studies have contributed significantly to the theoretical foundation of transdisciplinarity, its epistemological and methodological dimensions, its research potential and its practical applications.

Transdisciplinarity is simultaneously an attitude and a form of action (Klein, 2004: p. 521) that deals with problematic fields in such a way that it can: (a) grasp the complexity of problems; (b) take into account the diversity of life-world and scientific perceptions of problems; (c) link abstract and case-specific knowledge; and (d) develop knowledge and practices that promote what is perceived to be the common good (Pohl \& Hadorn, 2007, in: Leavy, 2011: p. 27)

Transdisciplinarity offers a different philosophy and approach both of how to effectively address issues/ problems and how to improve and change difficult situations we have been experiencing in the modern world, in different and multiple levels of reality and under conditions of complexity and uncertainty. Addressing these problems becomes precisely effective because of the way transdisciplinary achieves collaboration and integration among disciplines.

In Article 3 of the Charter of Transdisciplinarity (1994), De Freitas, Morin and Nicolescu state: "Transdisciplinarity complements disciplinary approaches. It occasions the emergence of new data and new interactions from out of the encounter between disciplines. It offers us a new vision of nature and reality. Transdisciplinarity does not strive for mastery of several disciplines but aims to open all disciplines to that which they share and to that which lies beyond them" (Nicolescu, 2002: pp. 148-149).

According to some scholars, transdisciplinarity does not seek for the unity of knowledge, in the sense that 
“...the development of a single unified 'truth' but rather, can seek to integrate the different knowledges by looking for coherence, correspondences and 'ridges' across the differences, generating knowledge by finding, identifying and communicating patterns across diverse disciplines and discourses" (Wickson et al., 2006: p. 1053).

We could argue that, in essence, transdisciplinarity generates and feeds an ongoing "dialectic synergy" between scientists and sciences in various forms and at many levels within a flexible and constantly evolving methodological framework; (see below 3). This dialectic synergy activates and motivates disciplines to co-create a "syn-epistemic wholeness", (see Figure 3) that is, a new "entity" that has a new philosophy, theory, epistemology, and new research strategies and practices offering enhanced potentials and alternatives to tackle complex and multi-parameter problems.

In this paper we will make a synoptic presentation of a Transdisciplinary Social Pedagogic Model, which emerged through extensive research projects carried out within school communities in Greece. The Model was based on many and high levels of collaboration, essentially within the multiple transdisciplinary synergies that incrementally and improvingly developed between: (a) the involved disciplines epistemologies and methodologies; and (b) all stakeholders (i.e. scientists, researchers, community partners, etc).

\section{The Transdisciplinary Social Pedagogic Model}

The general orientation of the research projects (Mylonakou-Keke, 2014, 2015)—within which the Trans- disciplinary Social Pedagogic ${ }^{1}$ Model was generated ${ }^{2}$ and which is now presented — was to address and more particularly to prevent pupils' antisocial behaviors. Children's antisocial behaviors, as manifested in different forms and in different places, are of great concern and at the same time are connected with the school, the family and the local community. The social pedagogic role of the school is shown to be as particularly important to address all forms of antisocial behaviors. The potential of this role is not limited only in schools but it can also be extended - through the projects that it itself can organize — to the utilization of multiple and dynamic links between the family and the community, especially in order to effectively address and prevent such kind of problems.

These projects were designed as "real world" problem-based research projects, their core was primary Greek schools with strong social pedagogic role and sought transdisciplinary synergies in various forms and at various levels (see below 2.5)

Each research project lasted nine months. Some schools chose to continue to operate their own project for the next year, by having been "autonomous": as school communities and utilizing the research experience they had acquired from their participation in the project.

\subsection{Problem Identification}

Antisocial behaviors — such as bullying, violence, victimization, the negative tackling of diversity, marginalization and exclusion of children by their fellow pupils inside and outside the school environment-have several parameters and are associated with various causes. The determining role of the school and the wider community in tackling and preventing various antisocial behaviors has emerged in a multitude of studies (indicatively: Farrington \& Ttofi, 2009; Georgiou, 2008; Kyriacou, 2003; Mylonakou-Keke, 2014, 2015; Olweus, 1993; Stephens, 2011). However, meta-analyses have shown that the effectiveness of many school-based projects is limited (Ferguson et al., 2007; Ryan \& Smith, 2009; Ttofi \& Farrington, 2011).

The key research questions that emerge are what and how can the school do so that efforts to address and prevent phenomena of antisocial behaviors can: (a) be successful; (b) have a lasting effect; (c) provide mechan-

\footnotetext{
${ }^{1}$ Social Pedagogy aims at personal and social progress and wellbeing, by educating individuals and groups from an early age so that they learn how to struggle throughout their lifetime in order to seek to improve and change existing life circumstances and assume substantial individual and collective - collaborative responsibility and action. It is an educational process in a broad sense, in and out of school that constantly takes into account the needs of individuals and groups that come primarily from social, cultural, economic and technological changes (Cameron \& Moss, 2011; Eichsteller \& Holthoff, 2012; Mylonakou-Keke, 2003, 2013; Petrie, 2011). Within this context, great importance is attached to strengthening the social pedagogic role of the school, via interconnections and collaborations that the school can utilize with the family and the community, especially to tackle problems, as well as opportunities that it can offer not only to the education of children and adolescents but also that of adults.

${ }^{2}$ The Transdisciplinary Social Pedagogic Model was generated by the author of the present paper. The Model has its roots in The Transdisciplinary Program SYNTHESIS (Kekes \& Mylonakou, 2006), and was formed and developed through ongoing research projects in school communities, which have been conducted more than a decade. The Model has been based on and at the same time has strengthened the social pedagogic role that some schools have developed as well as on the embedded transdisciplinary synergies in this specific social pedagogic role; see 2.4 below.
} 
isms that will discourage the onset of such behavior in the future; (d) systematically be backed up with sufficient knowledge of those directly and indirectly involved in the school community, who will form a strong collaboration network; and (e) can be able to address challenges, to curb potential barriers and overcome difficulties associated primarily with psychological and social factors?

\subsection{The Vision/Overall Aim and Main Objectives}

The vision and the overall aim of the research projects were originally the active participation and effective collaboration as many of those who directly or indirectly were involved in the school and in the wider community. Then, it was how to build a shared vision among the participants, which would seek the establishment, enhancement and utilization of a system of values, principles and beliefs, that is, a "social pedagogic ethos". This ethos will lead to a systemic transformation of the culture of all involved systems, that is, the school, the family, the wider school environment, the neighborhood and that of the community. This new culture and mentality can inspire the creation of a synergistic network between the stakeholders, in which there will develop strong mechanisms in school and the wider community that will prevent negative behaviors.

Whereas the general orientation of each research project was how to tackle and prevent antisocial behavior, it usually focused on one of these behaviors such as, for example, on bullying and violence or on diversity etc. (Mylonalou-Keke, 2014, 2015).

The main objectives for all stakeholders were to: (1) improve, strengthen, broaden and develop emotional, communication and social skills; (2) recognize and promote uniqueness and dignity of every human being, by strengthening self-respect, self-esteem, humility, self-control, self-efficacy and the interrelation between personal dignity and respect for the dignity of the "other"; (3) improve the perception of diversity/otherness and how to deal with it through positive interaction, mutual support and collaboration; (4) create favorable conditions for the integration of different types of knowledge that will facilitate the understanding of aspects of the problem and bring fruitful and creative ideas to address and solve it; (5) acquire experiential awareness of personal and, primarily, collaborative potential, and the multiplicative power that mutual trust and collaboration entail; (6) highlight and systematically promote a system of social pedagogic values and principles that resolutely prevent and respond to antisocial actions, and foster social norms that eschew, prevent and tackle negative behavioral tendencies towards others; and (7) to create a coherent and continuously expanding reciprocal network of mutuality, collaboration and multiple synergies, in which the school, the family, the neighborhood and the wider community will be involved.

\subsection{The Stakeholders}

As mentioned above, the Transdisciplinary Social Pedagogic Model pursues (and this is a benchmark and a criterion for success of the Model) the conscious and active participation and collaboration as well as willful stay in the current research project of as many as directly or indirectly involved in the school and the wider community, who are willing to participate. Given this situation, the stakeholders came from seven categories, and were: (a) academic researchers, experts, practitioners, coming from different disciplines, depending on the orientation of the research project and the requirements arising from the methodological practices during the course of the project. In line with this rationale, the participants of this group came, as appropriate, from disciplines such as: Social Pedagogy, Social Psychology, Education, Counseling, Systems Theory, Management, Theory of Literature, Theatre in Education etc.

The other categories of the stakeholders consisted of people who were directly or indirectly involved in schools and came from the school and the wider community, thus constituting a broad base of stakeholders. Those stakeholders were: (b) pupils who were enrolled in elementary schools in Greece; (c) educators, teachers and specialized teachers; d) school administrators; (e) all the staff involved with the school operation and life (i.e. cleaning staff, cafeteria owners, etc.); (f) parents, siblings and, generally, other members of the pupils' family, "significant others" for the pupils, who wished to participate in the project; (g) people from the local community, such as neighbors (who they may not have children of school age but were affected by the school life and even more by the antisocial behaviors manifested outside their houses), community leaders, responsible for educational matters, policy makers etc.

During the conduct of the research projects, every team consisted of stakeholders who came from each of the aforementioned categories, so that the team members can represent all the categories of stakeholders. 
The Transdisciplinary Social Pedagogic Model does not consider that the stakeholders belong to separate and individual layers, such as school, family, community, etc., and that should only be informed, be aware or even trained to know how-even with occasional collaborations-to deal with issues that concern them. It does not deal with them as recipients of policies and practices that were designed by others and they (the stakeholders) are invited to implement them. The Model considers that all stakeholders are partners, collaborators, co-researchers and co-creators, who themselves co-decide and determine which exactly the problem is that they wish to deal with. In line with this rationale, the orientation, the purpose and objectives of each project in each school community are co-formed by the stakeholders' collaboration.

In other words, the Model perceives stakeholders as interacting and collaborating systems that create a strong, collaborative and coherent network among them. As part of the network, these systems do continuous and mutual mentoring, manage knowledge, learn, co-create new integrated knowledge (see Figure 2) in order to address and resolve each problem that concerns them. Essentially, Model takes into account the existing levels, that is, school, family, community, and at the same time it goes beyond, by moving the focus away from the logic of a multi-level hierarchical structure towards the creation of a collaborative network dialectic.

\subsection{Key Features of the Transdisciplinary Social Pedagogic Model}

The central problem that should be addressed was the phenomenon of antisocial behaviors that pupils express in various ways inside and outside the school environment. In order for the key research questions (see 2.1 Problem identification) to be effectively answered it was necessary to develop and build a well-designed Trans- disciplinary Social Pedagogic Model.

At this point we should answer briefly two legitimate questions:

(a) Why is the Model considered Transdisciplinary?

(b) Why is the Model considered Social Pedagogic?

(A) Why is the Model considered Transdisciplinary?

There are certain key features that describe what constitutes the transdisciplinary character of a research, which we present in a generalized form, since we selected them from various studies (Gibbs, 2015; Leavy, 2011; Nicolescu, 1997; Wickson et al., 2006) and from our own related research efforts (Kekes \& Mylonakou, 2006; Mylonakou-Keke, 2013, 2014, 2015).

These seven key features of a transdisciplinary research are:

1) Transdisciplinary research is problem/issue-driven that manifests in the real world. The Model was created focusing on a serious problem that concerned the school community and should be treated efficiently, and that problem was the phenomenon of antisocial behaviors that pupils express inside and outside the school environment.

2) The problem is complex, multidimensional, with the different dimensions being heterogeneous. The phenomenon of antisocial behavior is complex, multifarious, has many parameters and is associated with various causes. Indeed, the high social ${ }^{3}$ and dynamic complexity of this the problem is related exactly to its many dimensions and heterogeneity (see footnote 2).

3) (as a consequence of the previous feature) To solve the problem the holistic approach, which combines the integration of different disciplines, epistemologies and methodologies, is necessary. We call it dialectic synergy between different disciplines, epistemologies and methodologies. The Model utilizes the dialectic synergies between Social Pedagogy and Systems Theory (more specifically, that of Human Activity System and Learning Organization), Knowledge Management, the Collaborative Action Research and the Syneducation Model; see Figure 1 and 2.5 .

\footnotetext{
${ }^{3}$ As far as social complexity is concerned, according to Adam Kahane (2004), a problem has high social complexity, when there are people involved in the problem who look at things very differently, depending on their values, assumptions, rationales and their objectives. In the case of antisocial behaviors, many different persons are involved directly or indirectly, and each one of them has a varying degree of involvement, his/her own principles, goals and needs, thus creating his/her own reality. As a consequence, there is differentiation on many levels; for example: children who repeatedly manifest antisocial behaviors lashing out against others (abusers), children who suffer these lashes to themselves (victims), children who are bystanders with a negative, or indifferent or sympathetic or compassionate attitude towards the victim, and the parents of these different children who often interpret their children's behaviors with different criteria and different principles and values. There is also differentiation among scientists who come from different disciplines and who approach the problem through their own interpretative scientific framework. According to Kahane, the dynamic complexity of a problem is related to the proximity the cause and the effect have in space and time. The impact of antisocial behaviors is often visible close in time when they occur, but, unfortunately, is visible much later for both the victim and the abuser [High dynamic complexity].
} 


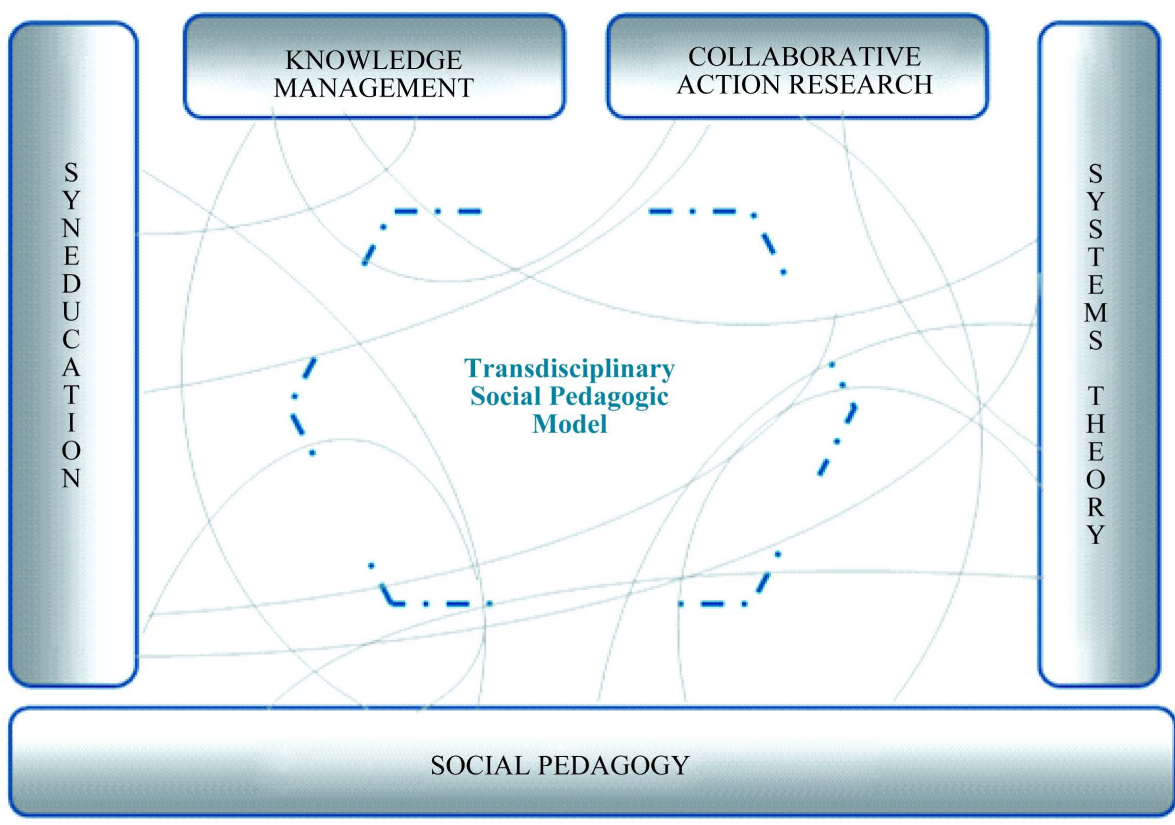

Figure 1. The transdisciplinary social pedagogic model.

4) The primary objective is to improve and change a problematic situation and solve a problem, through an evolving, dynamic methodology that is iterative and an ongoing part of the research process. As a discipline, Social Pedagogy was founded on the vision of action for improvement and changing educational and social problems through dynamic and participatory methodologies that are constantly redefined according to the process of problem solving; see 2.5. Moreover, given that these methodologies are fundamental characteristics of Systems Theory, the Collaborative Action Research and the Syneducation Model as well as the basic objective of Knowledge Management, new methodological practices are not only magnified and optimized but also-through integration and synergy - are created and utilized in the Model. These practices are not usually predetermined, but they are co-shaped and co-developed, while actions to deal with the problem are being taken, during the conduct of the research project.

5) The solution or improvement of the problem comes from the production of new knowledge that is a result of inter-related knowledge management processes and progression of learning. The Model is driven by this key feature (see 2.5), special interactive conditions are created (which was supported by the Syneducation model), such as experiential, interactive, mutual learning, creative and reflective thinking and collaborative management and cogeneration and transformation of knowledge; see Figure 2.

6) Dynamic interaction between theory and practice. Synergy and unity of theory and practice is a fundamental dimension of Social Pedagogy (see 2.5) and Systems Theory. In the Model, the stakeholders turned their values, principles, beliefs and thoughts into acts and then, through a critical, reflexive and interactive process, theoretized these practices, out of which new thoughts and perceptions emerged, which were based on values and principles and then these thoughts and perceptions turned into acts again. In other words, there was a continuous feedback and a mutual enrichment between theory and practice that was constantly improving. This improvement seemed not only to address the central problem but also the stakeholders' emotional, collaborative and social skills.

7) Many and high levels of collaboration between all stakeholders (including researchers) and the broader community are required. The Model (as mentioned earlier) sought to have a broad base of stakeholders, with a strong willingness to participate, who would consist of scientists, researchers, partners directly or indirectly related to the school and the wider community and who would be affected by the research project; see 2.3. Stakeholders become co-researchers, partners who develop multiple synergies among themselves and co-create new integrated knowledge to solve the problem, which they choose to identify.

It is worth emphasizing that many of the aforementioned key features of transdisciplinary research have already been fundamental principles or dimensions of disciplines, epistemologies and methodologies involved in 


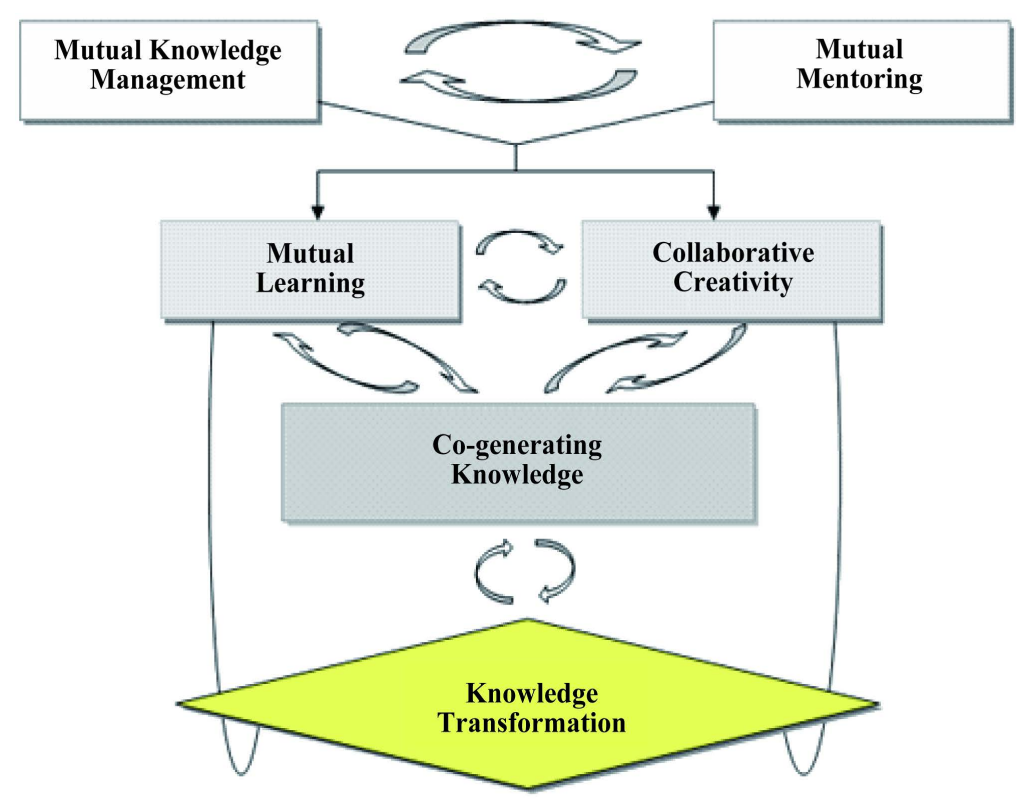

Figure 2. Transdisciplinary learning and knowledge.

the Transdisciplinary Social Pedagogic Model. In another words, the transdisciplinary character of a research is facilitated and supported effectively when disciplines, epistemologies and methodologies which are integrated in this research transdisciplinary dimensions or priorities or trends within themselves. And this happens because, when there is a transdisciplinary (or interdisciplinary) philosophical, theoretical and epistemological "predisposition" in a discipline, the dialectic synergy with other disciplines is considered a challenge, is facilitated, strengthened, expanded and is enriched.

(B) Why is the Model considered Social Pedagogic?

Social Pedagogy as a formal and institutionalized discipline has a history and presence of about two centuries. From its inception it has been based on the belief that social conditions can be influenced decisively through education and has had as its main objective the commitment of society to improve and change the educational and social situation. It has been connected with the dynamic address of issues/problems, thus contributing to the reform of social and education systems in Europe and the rest of the world (Kornbeck \& Rosendal Jensen, 2009; Mylonakou-Keke, 2013, 2014). Social Pedagogy is not limited to school life but places a strong emphasis on personal development, education, progress and well-being of all persons, throughout their lifetime.

We could define Social Pedagogy (Mylonakou-Keke, 2003, 2014) as the interdisciplinary field that "acts upon", and is a "functional mediator" between, human systems and their social, political, cultural, economic and technological hyper-system. Its role is particularly important for all people's adaptation, survival, growth, development, progress and wellbeing, throughout their lifetime. Social Pedagogy redefines the "chronotope" of education and its effectiveness is influenced, by and large, by the formation of a different "balance" between formal and the informal education, especially by the dominance of I.C. ${ }^{5}$, through which we have been experiencing a paradigm shift in Thomas Kuhn's (2012) terms.

There are some fundamental features of Modern Social Pedagogy that are its basic epistemological and methodological aspects and dimensions (Mylonakou-Keke, 2014, 2015) and that we are presenting them as follows:

1) Holistic-Systemic approaches: The complexity of the phenomena requires a holistic, systemic address of complex and often unanticipated issues that Social Pedagogy very often faces;

\footnotetext{
4"Functional mediator" refers to the meaning that the term has in Information and Communications Technology (I.C.T.), that is, an organized "mediation" between systems. In the case of Social Pedagogy, it is an organized "mediation" between the "bio-psychological-social system human" and the hyper-system that surrounds them; see analytically, Mylonakou-Keke, 2003.

${ }^{5}$ The I.C.T. has already created new reality for "vulnerable" or "disadvantaged" groups which results in new forms of social pedagogic issues, such as the "social digital exclusion”, lack of “digital opportunities” and “digital potential”, “digital divide”, “digital illiteracy”, “digital communalism”, cyber bullying, internet addiction etc. Thus, it is Social Pedagogy that should try to deal with this new harsh reality of the aforementioned forms of social pedagogic issues by utilizing different approaches from the traditional ones.
} 
2) Synergy and unity of theory and practice: A continuous interaction ${ }^{6}$ between theory and practice, in the form of a feedback loop is of utmost importance for Social Pedagogy, which is transformed into functional coexistence and, eventually, unites theory and practice.

3) Interdisciplinary character: Social Pedagogy generates strong interdisciplinary—and in some cases transdisciplinary-interconnections with many other disciplines, with all epistemological consequences that it entails and methodological parameters that it generates;

4) The continuous pursuit for change and improvement: The social pedagogic view wants individuals and groups to seek to make improvements and bring about changes in issues that concern them, while improving themselves;

5) The organized collective and collaborative action: The targeted improvement of and changes in issues are achieved by assuming personal and collective responsibility and taking organized collective and collaborative action; and

6) Prevention and intervention: Addressing social pedagogic issues effectively requires dynamic intervention in the field of action, while emphasizing the development of intervention for the generation of mechanisms to prevent the occurrence of social pedagogic problems.

As it becomes conspicuous, all the aforementioned fundamental principles and dimensions of Social Pedagogy are identical to the features of the transdisciplinary approach. In other words, Transdisciplinarity is not simply an "attitude" of Social Pedagogy, but the key features of the former are intertwined with the priorities of the latter. The transdisciplinary principles have already been embedded in Social Pedagogy and are essential elements of its identity, something that makes Social Pedagogy a discipline "familiar", "ready" and "experienced" to develop transdisciplinary synergies with other disciplines.

Concluding this section, we should note that the Model is based on the fundamental features of Social Pedagogy and Transdisciplinarity, and is called "Transdisciplinary Social Pedagogic" because this name: (a) implies the focus of the Model on the effective resolution (i.e. address and prevention) of problems (i.e. pupils' antisocial behaviors), which are at the same time interrelated educational and social problems; (b) highlights the transdisciplinary approach that is required due the special features that these problems have; (c) takes into account that this transdisciplinary approach is implemented in the "social pedagogic chronotope" (i.e. in the school, the wider community, in collaboration with people of different ages), and is both facilitated and enhanced by the values and the principles of Social Pedagogy; (d) embeds the transdisciplinary "predisposition" of Social Pedagogy, which integrates the fundamental dimensions of the transdisciplinary approach. This transdisciplinary "predisposition" of Social Pedagogy puts in motion and strengthens dialectical synergies with other disciplines, epistemologies and methodologies; see 2.5, below.

\subsection{The Implementation of the Social Pedagogic Model}

The Transdisciplinary Social Pedagogic Model was designed to motivate and utilize the multiple dialectic synergies among Social Pedagogy, Systems Theory (Learning Organization), Knowledge Management, the Collaborative Action Research and the Syneducation Model; see Figure 1. The Model deals with all disciplines and methods involved according to Nicolescu's following statement: ...the transdisciplinary method does not replace the methodology of each discipline, which remains as it is. Instead the transdisciplinary method enriches each of these disciplines, by bringing them new and indispensable insights, which cannot be produced by disciplinary methods. (Nicolescu, 2002: p. 122).

In the following paragraphs, we will provide (Mylonakou-Keke, 2015) briefly some information about these disciplines and methods [for Social Pedagogy, see 2.4 (B)].

Systems Theory (Checkland, 1995) approaches every system as a whole; it studies the interactive and interdependent connections of the parts of its subsystems and acknowledges that different properties from just the sum of the parts emerge from the whole. Generally, Systems Theory:

- provides mental "tools" to deal with complexity;

- professes the holistic approach $\kappa \alpha 1$ studies relations and interactions;

- seeks the unity of theory and practice;

- supports the organized collective and collaborative action;

${ }^{6}$ In a relevant discussion, in order to highlight the importance of the synergy of the theory and practice in Social Pedagogy (which leads to their unity), we provided a visualized transfer of Möbius strip, (with only one side) (Mylonalou-Keke, 2013). 
- considers necessary the researcher's involvement as part of the system in which the researcher carries out research in order to enhance the change of the system; and

- it accepts the response of the systemic model to reality as validation criterion of scientific knowledge. In the Transdisciplinary Social Pedagogic Model the stakeholders in each research project were considered as a "Learning Organization", according to Senge: ... where people continually expand their capacity to create the results they truly desire, where new and expansive patterns of thinking are nurtured, where collective aspiration is set free, and where people are continually learning how to learn together” (Senge, 2006: p. 3).

The Model utilized the five disciplines that Peter Senge identifies as:

- Personal Mastery is the discipline of personal growth and learning. People with high levels of personal mastery are continually expanding their ability to create the results in life they truly seek.

- Mental Models are deeply ingrained beliefs, mind-sets, assumptions and generalizations that influence how people understand the world and act.

- Building Shared Vision provides the focus and energy for learning and involves the skills of unearthing shared "pictures of the future" that foster genuine commitment and enrolment rather than compliance.

- Team Learning is the discipline that starts with "dialogue", the capacity of members of a team to suspend assumptions and enter into a genuine "thinking together".

- Systems Thinking is the fifth and cornerstone discipline that integrates the others, fusing them into a coherent body of theory and practice. This discipline helps people see how to change systems more effectively.

In order for the potential of the aforementioned disciplines in the research projects to be supported and maximized it was necessary that an intensive, iterative and with constant feedback collaborative research process, management and transformation of knowledge, accountability for improvement actions should be ensured. For these reasons, Knowledge Management, Collaborative Action Research and the Syneducation model were utilized in this Transdisciplinary Social Pedagogic Model.

Knowledge Management is a discipline (Kekes, 2007) that is used in business (Nonaka \& Tacheuci, 1995) and has recently been used in education (Sallis \& Jones, 2002).

The Transdisciplinary Social Pedagogic Model utilized the SECI model of Knowledge Conversion (Nonaka \& Takeuchi, 1995). The SECI model is based on four modes of knowledge creation and conversion: Socialization, Externalization, Combination and Internalization. These are created, in a "spiral" of knowledge, as a social process of continuous and creative interaction between tacit and explicit knowledge. The intention was that, through this model, opportunities could be created for each stakeholder's personal knowledge to be available to all the other stakeholders and, at the same time, team management and new knowledge production would be based on a process during which stakeholders:

- can share the ideas coming out of dialogue with others (the "socialization" of tacit knowledge);

- have an emergence of new ideas by utilizing (through research material, such as hypothetical scenarios etc., see below the educational material) analogy and metaphor (the "externalization" of tacit knowledge so that ideas can be turned into practice);

- combine knowledge to test their ideas (“combination” of explicit knowledge so that a more complete model of thinking and action than that of individual stakeholders is achieved); and

- develop new ideas and learn through action (“internalization” of explicit knowledge in ways particular to each stakeholder).

Collaborative Action Research especially with regard to education (Atweh et al., 1998; Checkland, 1995), is a participatory learning process, with iterative feedback processes that is conducted by the teams of stakeholders. Researchers become stakeholders, and the process of change itself becomes the subject of research. The stakeholders of each research project:

- begin by identifying a real-world problematic situation and which they wish to improve through the active intervention and action of all stakeholders;

- Then, an initial design-planning of actions is co-decided upon, which will help them as a team of stakeholders identify and define the aspects of the problematic situation and decide what action should be performed;

- what follows is: the undertaking of collaborative action, its systematic observation and the collection and recording of critiques, responses, impressions so that the consequences of this action for the enhancement and changes intended can be better understood; and

- All the aforementioned are utilized in the next stage of critical thinking and reflection on the action underta- 
ken and its results so that a new action plan could be redesigned and followed in the remaining stages of the research process.

Syneducation (synergy + education) is defined as a shared educational experience that is acquired simultaneously and in collaboration by people of different ages (representatives of the local community, policy makers etc.), different knowledge, experiences, interests and/or different social cultural backgrounds (Kekes \& Mylonakou, 2006; Mylonakou \& Kekes, 2005, 2007).

The Syneducation model is a systematic and collaborative learning process between people of different ages who function together in an interactive and collaborative learning environment where they organize and develop effective and proactive cooperative actions, based on Systems Methodology. Within this learning environment, the traditional roles of teaching and learning are abolished, whereas a co-dialectic and multiplicative dynamics of learning develops in which all participants both "teach" and "are taught".

Within the Syneducation model, stakeholders—receiving a simultaneous and interactive, collaborative education:

- seek to address and manage collaborative situations where they co-decide upon and consider them important or critical;

- operate with mutual influence, guidance and mutual mentoring, despite age difference, utilizing children's creativity, freshness and resilience which are fed by and at the same time feed adults with interaction and synergy;

- start from their own vision so as to create their shared vision for the issue that concerns them, designing an ameliorative change with their active participation in and commitment to this issue, trying to involve as many stakeholders as possible;

- encourage creative interaction and collaborative learning;

- utilize personal and communication skills and experiences, encouraging the development of new creative ideas;

- strengthen personal and collective responsibility, by undertaking substantive actions for collaborative prevention and intervention;

- study and evaluate their collective practices, behaviors and actions; they re-examine their personal beliefs and their consequent effects on their interpretation codes and alter/enhance their perceptions and attitudes; and

- gradually increase the degree of involvement and participation in the project and seek to operate as multipliers, attracting other people to the project; in this way, they seek to create a strong and ever-growing network of interaction, in which they produce and develop social capital that further enhances the fulfillment of their shared vision.

It is conspicuous that the fundamental principles and dimensions of Social Pedagogy pertain and get in harmony with basic key points from each discipline and method that participates in the Transdisciplinary Social Pedagogic Model and, of course, with the key features of transdisciplinary research. This facilitates and strengthens transdisciplinary synergies that are generated between them.

In the Transdisciplinary Social Pedagogic Model ongoing and multiple transdisciplinary synergies among disciplines and methods that mentioned earlier have been developed, synergies that reach a "harmonic synchronization". The iterative way of this dynamic and evolving methodology entails a "transdisciplinary spirit" which invigorates all the research process and enables it to establish a new paradigm, this new research Model we are proposing.

According to the function of the Model, while these research projects were being conducted (MylonakouKeke, 2014, 2015), strategies, methodological practices and techniques were utilized that supported effectively the aforementioned dynamic and evolving methodology and were seeking to fulfill the collectively identified (by the stakeholders) objectives and purposes of the respective research project. Emphasis was placed on developing experiential team activities favoring the necessary interactive and collaborative learning environments for the given methodology.

The function of the Model was based on iterative and ongoing cycles/phases that were seeking to improve stakeholders' transdisciplinary learning and knowledge every time through experiential team activities that utilized educational material.

Transdisciplinary learning and knowledge, which derived from mutual knowledge management, mutual mentoring, collaborative creativity, mutual learning and cogenerating knowledge that was leading to a continuous transformation of knowledge; see Figure 2). 
The experiential team activities were based on the utilization of creative strategies, techniques, practices and tools, such as role playing, the Jig Saw techniques (Aronson et al., 1978), cycles of change (Macfarlane \& Cartmel, 2012), six thinking hats (DeBono, 2009), SCAMPER (Sloane, 2006) questions (customized for the needs of the projects), dilemma situations, scenario-building, metaphors, analogies, brainstorm, conceptual maps etc.

The educational material that was created and utilized in the research projects consisted of hypothetical scenarios, stories, storytelling and fairy tales, images and illustrated stories with multiple possibilities of reading, directed incidents, simulations of various situations, videos, films, songs, artwork etc. Much of the educational material offered the opportunity to continue and conclude the story through sequential problem settings, decision making and problem solving.

The educational material highlighted situations related to various forms of antisocial behaviors (focused on the specific problem that was central to each project) that were manifested and dealt with in many different ways. Every time, these ways showed different and interrelated sides of difficult and problematic situations, such as (indicatively): (a) how and to what extent prejudices, stereotypes, mind-sets, beliefs, assumptions $\kappa \alpha l$, generally, the personal interpretation code influence behaviors and actions; (b) how many forms of diversity there are and how unique and valuable each person is; (c) how emotions and experiences guide a person's behaviors; (d) how the presence or the absence of emotional, communication, collaboration and other social skills of each participant in every alleged situation influence him/her; (e) the potential options the participants have to deal with the problem from a social pedagogic perspective; and (f) the role of endorsing values and principles, adopting rules as well as establishing and maintaining boundaries.

Being based on the aforementioned function of the Model and its transdisciplinary methodology and having utilized the educational material, each stakeholder gradually came to: (a) experience actual situations that highlighted antisocial behaviors; (b) internalize his/her experience and new knowledge; (c) re-examine his/her behaviors and attitudes through empathic thinking; (d) "search for once again" and "see again" himself/herself through new patterns of behavior; (e) expand and redefine his/her perceptions; (f) re-examine his/her boundaries, values and his/her interpretation code; (g) become aware of the enrichment of his/her potential and skills-by interacting with others, mutual mentoring, mutual learning, collaborating and becoming aware of the multiple possibilities of the team; and thus (h) each stakeholder was led to bring out and strengthen, at an individual and team level, a code of values that would guide his/her behavior and would compose a "social pedagogic ethos" and ultimately a culture that would define the function of the school community.

According to the function of the Transdisciplinary Social Pedagogic Model, at every cycle/phase in each research project, during the generation and transformation of knowledge there was a high level of personal and collective reflection and increasing personal commitment, the target of which was to plan the next enhanced actions. Using this dynamic and evolving methodology, the iterative way of creative, reflective and critical thinking, as well as that of learning and action entails the simultaneous enhancement of stakeholders' skills and modeling of new behaviors. These behaviors lead to changes in stakeholders' way of thinking and to the emergence and strengthening of values and the formulation of a new (social pedagogic) "culture".

During the function of the Model a factor that was given special importance was each stakeholder's gradual transformation of personal involvement through the change of perceptions, attitudes and behaviors and adoption of new roles. The dynamics of the Model so that each stakeholder's involvement in the transdisciplinary research project was gradually becoming more and more substantial; the Model supported a gradual move from a situation that one could have been indifferent towards a more active, creative and collaborative participation. This transformation of personal involvement brought an interactive change in each stakeholder's perceptions, attitudes and behaviors (at each one's personal level).

The function of the Model has shown [as previous transdisciplinary research projects had shown (Kekes, \& Mylonakou, 2006)], that there is a spectrum of transformation in stakeholders' involvement. This spectrum usually started from an initial state (even before the stakeholder's participation in the research project), during which the stakeholder could be Indifferent—Disinterested (Uninvolved), s/he was gradually becoming an Observer, a Participant, a Collaborator, a Co-creator, a Critical Analyst, and s/he finally may have ended up being a Multiplier (who was supported especially by Syneducation Model). Acting as a multiplier, the stakeholder felt the need to communicate, disseminate and share with others the knowledge and experiences s/he acquired, and, in addition, to involve other people in the whole process. 


\section{Transdisciplinarity or Syn-Epistemic Wholeness}

According to the Transdisciplinary Social Pedagogic Model, multiple transdisciplinary synergies were being developed throughout the research. These synergies were dialectic synergies among disciplines, epistemologies, methodologies, research strategies, practices, ways of learning, knowledge and among scientists, researchers and all other stakeholders; see Figure 3.

By using the term Dialectic Synergy, we should note that:

The word Dialectic ${ }^{7}$ includes the concepts: (a) the dynamic $\kappa \alpha$ interaction [between elements that compose a system or a particular situation]; and (b) the fruitful synthesis even of opposite perceptions, views and arguments.

The word Synergy ${ }^{8}$ is comprised of the concepts of: harmonious collaboration, mutuality, coordination of synchronization, common interest and dynamic state. The dialectic synergy involves the dynamics among continuous interactions and collaborations, among situations, such as: Functional interaction, Ongoing feedback, Mutual reinforcement, Co-creation, Collaborative action and "Co-evolution” (Co-development); see Figure 3.

The dialectic synergies taking place in the Transdisciplinary Social Pedagogic Model result in Syn-epistemic Wholeness. This term consists of: (a) the compound word "syn-epistemic”, whose prefix syn [a Greek preposition meaning "along with" and is mainly used as a first component of compound words (e.g. synthesis)] and the

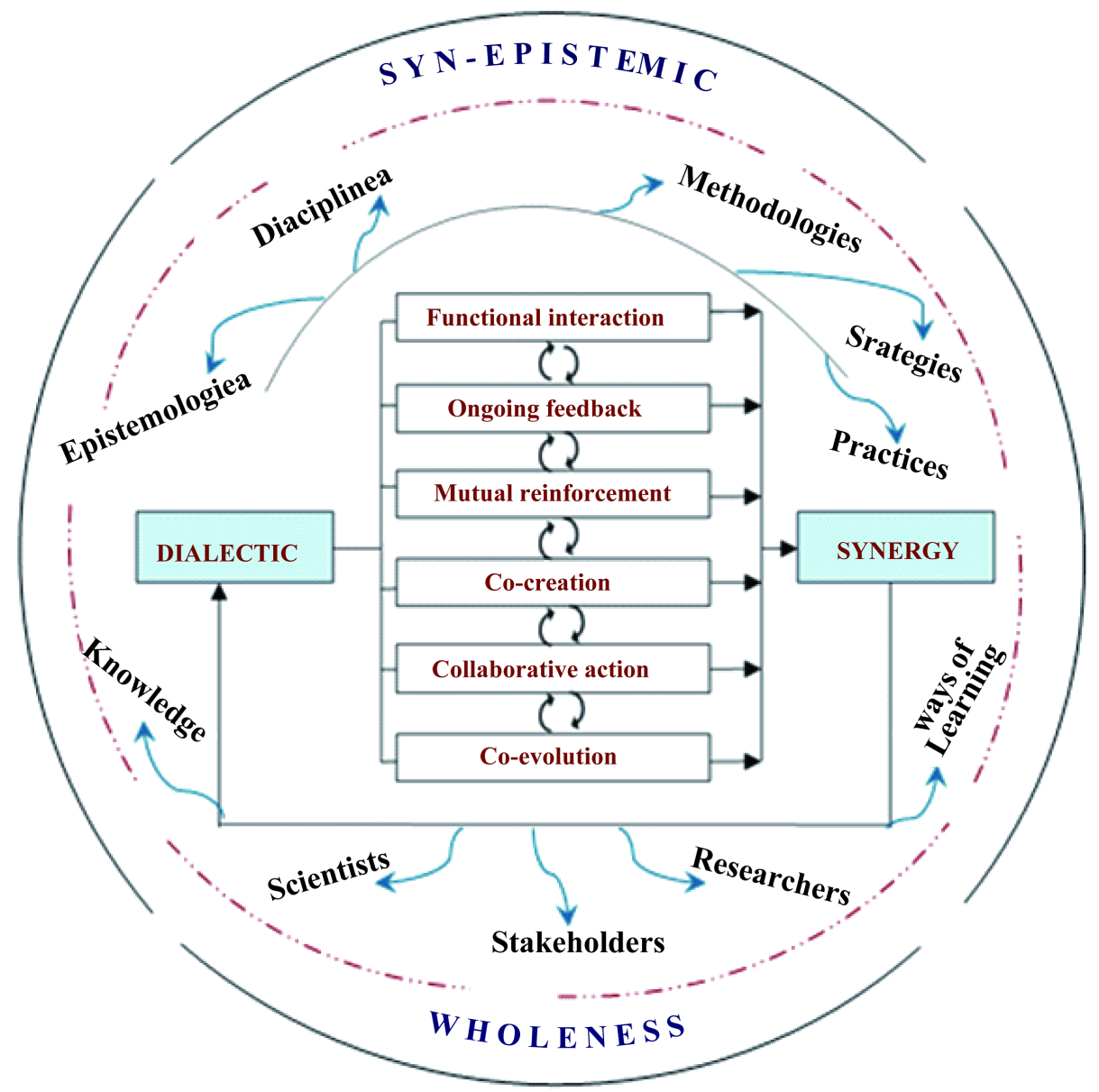

Figure 3. Syn-epistemic wholeness emerged from dialectic synergy.

\footnotetext{
${ }^{7}$ The word dialectic comes from the Greek word $\delta \iota \alpha \lambda \varepsilon \kappa \tau \imath \kappa \eta$ (dialektike) that means the art of argument through interactive questioning and answering that arrives at the truth by the interaction and the synthesis of logical arguments. It is an important element of ancient Greek philosophy (Socrates and Plato, Aristotle, Zeno of Elea).

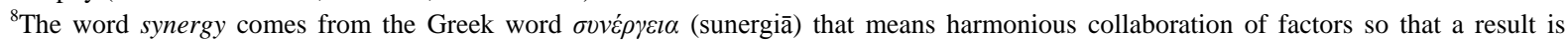
achieved; this result is very important for all participants and could never be achieved simply by the sum of the results of the individual actions and factors.
} 
adjective epistemic, [the Greek adjective epistēmikós derives from the Greek word epistếm(ē): knowledge, science], thus relating knowledge to science; and (b) the noun wholeness, that means an entirety, a whole which, according to ancient Greek philosopher Aristotle "the whole is greater than the sum of the parts" (Metaphysics, H 6).

From a more general view, Syn-epistemic Wholeness is generated by the constant dialectic synergy among disciplines and among scientists. Syn-epistemic Wholeness is a new "entity" that transcends the involved disciplines (or sciences) without replacing them; it rather enriches them by offering the potential of a synchronous, harmonic function in a flexible and constantly evolving methodological framework. By this logic, the Syn-epistemic Wholeness allows the emergence of a new general perception, the essence of a new "paradigm", which has new philosophy, theory, epistemology as well as new research strategies and practices and which offers rich possibilities and alternatives to address complex, multi-parameter and multifarious problems.

In this train of thought, we could propose that the term Synepistemic Wholeness could be used instead the term Transdisciplinarity.

\section{Evaluation}

The evaluation of the Transdisciplinary Social Pedagogic Model was made through the evaluation of each research project and by a team of specialists who evaluated the effectiveness of the Model with additional criteria.

While every research project was being evaluated (Mylonakou-Keke, 2014, 2015), all the stakeholders in the respective project participated as evaluators. The evaluation was conducted through a combined evaluation model $^{9}$. That provided formative evaluation in order to highlight areas that needed improvement while the research project was being conducted and a summative evaluation. This model of evaluation utilizes many of the processes and procedures of empowerment participatory evaluation.

Additionally, the model includes specialized evaluations and self-evaluations, by utilizing tools such as selfobservation keys, writing diaries, reports, reviews, protocols, the "Most Significant Change" Technique (Dart \& Davies, 2003), questionnaires, scales, SWOT analysis (Pahl, \& Richter, 2007), evaluation grids with a variety of criteria and indicators (such as collaboration indicators), etc. that were utilized by the stakeholders according to their age, and were customized to their age capabilities.

The summative evaluation was made by all teams of stakeholders: (a) academic researchers, experts, practitioners; (b) pupils; (c) educators, teachers and specialist teachers; (d) school administrators; (e) the rest of the school staff; (f) parents and members of the pupils' family etc.; (g) local community members, policy makers etc.; for more details, see 2.3.

There were categories of evaluation criteria that were common to stakeholders, and there were categories that were different for each team of stakeholders. Those categories were allocated to questions which aimed to lead stakeholders, through a reflective process, to evaluate different dimensions of their experience of participating in the research project and ultimately to state to what extent the aims and objectives of the project had succeeded.

In the final stage of each research project, in order to deduct and formulate final conclusions, all the teams of stakeholders/evaluators participated in a dialectic debate and processed their experience throughout the research project.

\section{Outcomes/Advantages}

Ithaka gave you the marvelous journey.

Without her you would not have set out.

She has nothing left to give you now.

And if you find her poor, Ithaka won't have fooled you.

Wise as you will have become, so full of experience, you will have understood by then what these Ithakas mean.

Constantine P. Cavafy ${ }^{10}(1863-1933$, Greek poet)

\footnotetext{
${ }^{9}$ The combined evaluation model was based partly on evaluation procedures that were used in other transdisciplinary research projects (Kekes \& Mylonakou, 2006; Mylonakou-Keke, 2013). Additionally, a variety of specialized evaluation keys and indicators were constructed for the evaluation of different aspects of the research projects (some of which have yet to be published).

${ }^{10}$ Cavafy, C.P. (1992). Collected Poems, translated from the Greek, by Edmund Keeley and Philip Sherrard and edited by George Savidis. Revised Edition. Princeton, New Jersey: Princeton University Press.
} 
Our research "journey" in the deep, unpredictable and often hectic "sea" of the life of the school community never ends. The "ship", the Transdisciplinary Social Pedagogic Model, seems to have reached its destination, Ithaca (i.e. address and prevention of antisocial behavioral problems). However, what has this journey resulted in?

The combined system of evaluation provided a number of findings at many and different levels. Within the context of this paper it is impossible for us to present the aggregate or individual results of each stage of the evaluation of each research project (Mylonakou-Keke, 2014, 2015) or to present the many different potentials of Transdisciplinary Social Pedagogic Model, as highlighted by the evaluation.

The response of the Model to transdisciplinary and social pedagogic character has already been presented in 2.4 (Features of the Transdisciplinary Social Pedagogic Model).

In the following sections, we will refer indicatively and briefly to some general outcomes that were evaluated as very positive; these outcomes are presented in categories, which are at the same time the great advantages of this Model. These categories of outcomes/advantages, of course, interact with each other, are interrelated and are the following:

A) The symphony of diversities

One important outcome of the projects was stakeholders' awareness that each person has his/her own diversity, which comes from his/her uniqueness, which is important but requires continuous enhancement efforts (social pedagogic ${ }^{11}$ perception of diversity).

The stakeholders (were people of different age and came from different educational cultural backgrounds) became aware that their initial perception of the diversity of the "other" is directly linked with their emotional patterns, their perceptions and underlying representations and their interpretation code, which are greatly influenced by their cultural and social experiences and guide their behaviors.

The Model helped so that not only persons' diversities — without effacing their uniqueness—but also those of evolving disciplines, methodologies and strategies can be synthesized in a "harmonic polyphony" and often resulted in "symphony". As far as people are concerned, this "symphony" was diffused by the stakeholders in the school community environment and prevented the appearance of a dissonant behavior.

B) Mutuality, Collaboration, Dialectic Synergies

There was a continuous "blooming" of dialectic synergies at different levels. The Model motivated and reinforced continually dialectic interaction, mutuality and collaboration at many and high levels, thus fostering dialectic synergies that incrementally and improvingly developed among the involved disciplines epistemologies, methodologies, research strategies, practices, ways of learning, knowledge, as well as among all stakeholders.

Those synergies "blossomed" and developed in an interactive, collaborative environment that was characterized by mutual influence, mutual mentoring (despite the difference in age ${ }^{12}$ groups, the syneducation environment helped a lot) and mutual learning. Mutual learning was also supported by a feedback loop between "we search by learning"- - discovering, creating, organizing and developing personal and collective knowledge-and "we learn by searching”, which generated new knowledge and continuous transformation (see Figure 2).

C) Integration and transformation

The dialectic synergies created different levels and degrees of integration $\kappa \alpha 1$ transformation, such as those among methodologies, research strategies, practices, learning and knowledge from different disciplines and different people. Within this context, a dynamic collaborative, a "synergistic" environment was generated, in which special experiential, interactive and collaborative learning conditions developed; these conditions favored participants' collaborative knowledge, creation, and conversion (which was supported by the SECI model) and improved their abilities of knowledge transformation, through the integration of the aforementioned methodologies; (see 2.5).

Eventually, the Model was creating and favoring such conditions that everyone was learning from each other

\footnotetext{
${ }^{11}$ Social Pedagogy seeks not only to make people aware of the issue of diversity/otherness but also to educate them so that they can have an attitude of acceptance and coexistence rather than an attitude of tolerance, or compassion or superiority towards the "other". Social Pedagogy is not confined to the perception of social adaptation and integration of those people who are "different" or experience some sort of disability or marginalization of any kind. Social Pedagogy believes that each person is different and one of its fundamental principles is to make all people unleash their own potential, ensure this diversity through unity and motivating people to achieve improvement, change and transform an existing situation, mainly through personal and collective responsibility and cooperative action. In brief, Social Pedagogy perceives each person's diversity as otherness, something that springs out of the person's uniqueness (Mylonalou-Keke, 2013).

${ }^{12}$ The logic of the linear transmission of knowledge and experience from the older to the younger generation was not acceptable in this Model.
} 
and improved from one another. All this process led to what could implicitly be stated by Bacchylides of Ceos, one of the Greek lyric poets (518 BC-452 BC): "From ancient times up to now, [it is known that] one becomes wise from the other." (Paean., fr. 5 Maehler).

D) Multiple and enriched skills and relations

The emotional, communication and social skills [present or absent in various forms, as they were projected on and highlighted in the educational material], through the process of knowledge creation and transformation, were to be "taught" (in a broad sense) and be cultivated by the stakeholders through experiential, collaborative and creative learning. This led to an ongoing and dynamic cogenerating and evaluation of knowledge, experiences and practices and recreation of all those parties involved, through intellectual, emotional and social interactions of the stakeholders, which eventually broadened and enriched their own cognitive, emotional, collaborative and social skills and improved their relationships. These helped significantly so that any psychological barriers that initially were raised by the stakeholders would be overcome.

It became conspicuous that the Model helped so that authentic relationships between the stakeholders can be progressively built. Such relationships start from a personal and a shared vision and from a sense of mission and become authentic relationships based on mutuality, trust, intimacy, genuine interest in the other, cooperation with each other, a sense of duty and the assumption of personal and collective responsibility for collaborative action, resulting in the production of "social capital" and the creation of a synergistic network between the stakeholders.

E) Creativity and Innovation

Creativity and innovation were inherent in each transdisciplinary research project, were the foundations of each approach while the project was being conducted. Creativity and innovation are very important advantages of the Model, which offered an ongoing "oxygenation" and made all the progress of research process alive and, of course, participated in all aforementioned categories of research outcomes. Creativity inspired and encouraged experimentation, contributed significantly to the birth of unpredictable ways of thinking and innovative ideas that facilitated the achievement of the aim and the objectives of each project. Among all other things, all stakeholders noted that creativity fueled the very positive psychological climate that prevailed (which was reinforced by children's spontaneity), interactive humor, team spirit, motivation for participation, the sense of shared mission as well as team learning and action, and affected the quality of the team function and ultimately the effectiveness of the project.

F) Response to the objectives, Usefulness and Ethical background

The evaluation showed that the Transdisciplinary Social Pedagogic Model was of crucial importance for the success of the projects. The Model helped the stakeholders reconsider and re-organize gradually and progressively (depending on every one's knowledge transformation abilities and level of understanding), previous opinions on and their perceptions of various forms of antisocial behavior; it helped them experience new behaviors and potentialities. Thus, they were led to extensive interpretive schemes and gave a new meaning to various terms and conditions, such as: diversity, uniqueness, the need for human cohabitation and communication, forms of and potential for cooperation, taking personal and collective responsibility as well as collaborative actions so that a negative situation could be changed and enhanced.

In essence, the Model helped the stakeholders to redefine and reinforce principles and a code of values and to incorporate them into practices, within a social pedagogic framework, which eventually transform and enhance the culture of the school and the wider community. It is about building and strengthening of what we call "social pedagogic ethos". Social pedagogic ethos (Mylonakou-Keke, 2015) creates a philosophy that translates into a way of life and consists of core values and principles (such as love, solidarity, justice, magnanimousness, self-respect, a respect for every person's dignity, empathic understanding, taking personal responsibility, continuous effort for self-improvement, selflessness, diligence, cooperativeness, democratic principles). The utilization of the Model has shown that the social pedagogic ethos can gradually build and consolidate the school community and then get diffused, thus guiding all school life and eventually influencing the wider community.

G) Dissemination and the duration of the impact of the outcomes

During the function of the Model (as mentioned in 2.5), the spectrum of transformation of the stakeholders' personal involvement reached for many stakeholders to the multiplier state. In this state the stakeholders felt a strong desire to communicate and disseminate both their experiences and the knowledge they had acquired to third parties as well as to involve other people in the same or in similar subsequent projects. This dissemination facilitated the penetration of the impact of the research project on the local community. 
Another important and useful outcome of the Transdisciplinary Social Pedagogic Model has been the duration of the participant's systemic transformation that was attained in the culture of the school and the wider community, especially in motivating people against antisocial behaviors. It is worth noting that in one of these projects, six months after that project was completed, an additional evaluation took place so we could see what had remained of that project. According to this second evaluation, all former stakeholders felt that they were in an ongoing and important mission and that feeling pleased them and at the same time made them feel responsible.

Having been "educated" by the Model, the stakeholders knew ways of healthy and effective responses in cases of negative behavior event. The pupils, who were some of the stakeholders, had enriched their skills and reconsidered their values, perceptions and behaviors, so they stopped expressing antisocial behaviors. Instead, they expressed very positive behaviors on many levels, which were enhanced and fed back by the interaction with the stakeholders who, in this case, were their own parents and other pupils' parents. In case that there were some pupils with tendencies for antisocial behaviors, those pupils were aware of the fact that the culture of the school did not allow these behaviors and that they would meet strong and organized opposition from everyone.

Finally, the Model has brought up advantages that helped so that the objectives of the transdisciplinary research project could be achieved to a great degree and many other factors and parameters that had a positive impact on the individual and collective level of the life of the school and the wider community could be enhanced.

The weaknesses of the Model are that: (a) it requires that coordinators should be continuously present in stakeholders' teams (as collaborator and co-researcher) and should have good knowledge of Transdisciplinary research methodology, as well as of the methodologies of disciplines involved in the Transdisciplinary Model; and (b) several hours beyond those spent during formal school operation were required as well as time commitment of different categories of the stakeholders.

\section{Instead of an Epilogue}

To go back to Cavafy, finally it seems that the travelers (the stakeholders) have enjoyed the voyage (transdisciplinary research), have learned and gained a lot from it.

Hope the voyage is a long one.

May there be many a summer morning when,/with what pleasure, what joy, you come into harbors seen for the first time; may you stop at Phoenician trading stations/to buy fine things, mother of pearl and coral, amber and ebony, sensual perfume of every kind-las many sensual perfumes as you can; and may you visit many Egyptian cities/to gather stores of knowledge from their scholars...

With so many goods they have brought together and so many skills they have acquired, the travelers learned how to face effectively any difficulties (Model's weaknesses).

Laistrygonians and Cyclops,/angry Poseidon-don't be afraid of them:

you'll never find things like that on your way/as long as you keep your thoughts raised high, as long as a rare excitement/stirs your spirit and your body.

Laistrygonians and Cyclops,/wild Poseidon-you won't encounter them unless you bring them along inside your soul, /unless your soul sets them up in front of you.

In any case, throughout the voyage a fair wind was blowing, and that was the Transdisciplinary Social Pedagogic Model, which was generating a transdisciplinary spirit, that constantly facilitated the course of the ship.

The travelers seemed to have been rejoicing the voyage without forgetting where they wanted to go (address and prevention of antisocial behavior)

Keep Ithaka always in your mind./Arriving there is what you are destined for.

And so they arrived in Ithaca, everyone carrying with him/her what could be gathered from the voyage...

wealthy with all you have gained on the way,

But this Ithaka is a new starting point for new journeys. All "travelers”, the stakeholders—enriched with ex- 
periences and knowledge-are ready to choose a new Ithaca, and start, if they want to, a new journey, since they have now acquired "transdisciplinary research seamanship" to do so. Each new destination from now on will not be any Ithaca but the journey itself.

\section{References}

Aronson, E., Blaney, N., Sikes, J., Stephan, G., \& Snapp, M. (1978). The Jigsaw Classroom. Beverly Hills, CA: Sage Publications.

Atweh, B., Kemmis, S., \& Weeks, P. (Eds.) (1998). Action Research in Practice: Partnerships for Social Justice in Education. London \& New York: Routledge. http://dx.doi.org/10.4324/9780203268629

Cameron, C., \& Moss, P. (2011) (Eds.). Social Pedagogy and Working with Children and Young People: Where Care and Education Meet. London and Philadelphia: Jessica Kingsley Publishers.

Cavafy, C.P. (1992). Collected Poems (Revised Edition). Translated by Edmund Keeley and Philip Sherrard. Edited by George Savidis. Princeton, NJ: Princeton University Press.

Checkland, P. (1995). Systems Thinking, Systems Practice: Includes a 30-Year Retrospective. Chichester \& New York: John Wiley and Sons Ltd.

Dart, J., \& Davies, R. (2003). A Dialogical, Story-Based Evaluation Tool: The Most Significant Change Technique. American Journal of Evaluation, 24, 137-155. http://dx.doi.org/10.1177/109821400302400202

DeBono, E. (2009). Six Thinking Hats. Penguin Books.

Eichsteller, G., \& Holthoff, S. (2012). The Art of Being a Social Pedagogue: Developing Cultural Change in Children's Homes in Essex. International Journal of Social Pedagogy, 1, 30-45.

Farrington, D. P., \& Ttofi, M. M. (2009). School-Based Programs to Reduce Bullying and Victimisation. Campbell Systematic Reviews, 2009:6. http://dx.doi.org/10.4073/csr.2009.6

Ferguson, C. J., San Miguel, C., Kilburn, J. C., \& Sanchez, P. (2007). The Effectiveness of School-Based Anti-Bullying Programs: A Meta-Analytic Review. Criminal Justice Review, 32, 401-414. http://dx.doi.org/10.1177/0734016807311712

Georgiou, S. N. (2008). Parental Style and Child Bullying and Victimization Experiences at School. Social Psychology of Education, 11, 213-227. http://dx.doi.org/10.1007/s11218-007-9048-5

Gibbs, P. (Ed.) (2015). Transdisciplinary Professional Learning and Practice. Dordrecht: Springer.

Hirsch Hadorn, G., Biber-Klemm, S., Grossenbacher-Mansuy, W., Hoffmann-Riem, H., Joye, D., Pohl, C., Wiesmann, U., \& Zemp, E. (2008). The Emergence of Transdisciplinarity as a Form of Research. In: G. Hirsch Hadorn et al. (Eds.), Handbook of Transdisciplinary Research (pp. 19-39). Heidelberg: Spinger. http://dx.doi.org/10.1007/978-1-4020-6699-3

Kahane, A. (2004). Solving Tough Problems: An Open Way of Talking, Listening, and Creating New Realities. San Francisco, CA: Berrett-Koelher Publishers.

Kekes, I. (2007). Knowledge Management within the Modern Technological Environment. Athens: Diadrasis Editions. (In Greek)

Kekes, I., \& Mylonakou, I. (2006). Syneducational Transactions among Students, Parents, Educators and Policy Makers: The Transdisciplinary Program SYNTHESIS. Interactive Learning Environments, 14, 35-54. http://dx.doi.org/10.1080/10494820600711496

Klein, J. T. (2004). Prospects for Transdisciplinarity. Futures, 36, 515-526. http://dx.doi.org/10.1016/j.futures.2003.10.007

Klein, J. T. (2008). Evaluation of Interdisciplinary and Transdisciplinary Research: A Literature Review. American Journal of Preventive Medicine, 35, S116-S123. http://dx.doi.org/10.1016/j.amepre.2008.05.010

Klein, J. T. (2010). A Taxonomy of Interdisciplinarity. In: R. Frodeman, J. T. Klein, \& C. Mitcham (Eds.), The Oxford Handbook of Interdisciplinarity (pp. 15-30). Oxford: Oxford University Press.

Kornbeck, J., \& Rosendal Jensen, N. (Eds.) (2009). The Diversity of Social Pedagogy in Europe. Bremen: Europäischer Hochschulverlag.

Kuhn, T. S. (2012). The Structure of Scientific Revolutions (50th Anniversary Edition). Chicago, IL: University of Chicago Press. http://dx.doi.org/10.7208/chicago/9780226458144.001.0001

Kyriacou, C. (2003). Helping Troubled Pupils. Cheltenham: Nelson Thornes.

Leavy, P. (2011). Essentials of Transdisciplinary Research: Using Problem-Centered Methodologies. Walnut Creek, CA: Left Coast Press.

Macfarlane, K., \& Cartmel, J. (2012). Circles of Change Revisited: Building Leadership, Scholarship and Professional Identity in the Children's Services Sector. Professional Development in Education, 38, 845-861.

http://dx.doi.org/10.1080/19415257.2012.680603 
Mylonakou, I., \& Kekes, I. (2005). Syneducation (Synekpaidefsis): Reinforcing Communication and Strengthening Cooperation among Students, Parents and Schools. Harvard Family Research Project, Cambridge, MA: Harvard Graduate School of Education. http://www.hfrp.org/content/download/1262/48765/file/syneducation.pdf

Mylonakou, I., \& Kekes, I. (2007). School, Family and the Community in Cooperation: The Model of Syneducation. International Journal about Parents in Education, 1, 73-82.

Mylonakou-Keke, I. (2003). Issues of Social Pedagogy. Athens: Atrapos Editions. (In Greek)

Mylonakou-Keke, I. (2013). Social Pedagogy: Theoretical, Epistemological and Methodological Dimensions. Athens: Diadrasis Editions. (In Greek)

Mylonakou-Keke, I. (2014). Chapter 8: Transdisciplinary Approaches to Education in the Time of Crisis. The Case of Addressing Bullying and Violence in Schools. In Psychological and Educational Approaches in Times of Crisis: Exploring New Data (pp. 135-158). New York: Untested Ideas Research Center.

Mylonakou-Keke, I. (2015). Social Pedagogy and School Community. Preventing Bullying in Schools and Dealing with Diversity: Two Sides of the Same Coin. International Journal of Social Pedagogy, 4, 69-89.

Nicolescu, B. (1997). The Transdisciplinary Evolution of Learning. In Proceedings of the International Congress on What University for Tomorrow? Towards a Transdisciplinary Evolution of the University, Locarno, 30 April-2 May 1997, 1-11. http://www.learndev.org/dl/nicolescu_f.pdf

Nicolescu, B. (2002). Manifesto of Transdisciplinarity. Albany: State University of New York Press.

Nonaka, I., \& Takeuchi, H. (1995). The Knowledge Creating Company: How Japanese Companies Create the Dynamics of Innovation. New York: Oxford University Press.

Olweus, D. (1993). Bullying at School: What We Know and What We Can Do. Cambridge, MA: Blackwell.

Pahl, N., \& Richter, A. (2007). Swot Analysis_Idea, Methodology and a Practical Approach. Munich: GRIN Verlag.

Petrie, P. (2011). Communication Skills for Working with Children and Young People: Introducing Social Pedagogy. London and Philadelphia, PA: Jessica Kingsley Publishers.

Prigogine, I., \& Stengers, I. (1984). Order out of Chaos: Man’s New Dialogue with Nature. Toronto: Bantam Books.

Ryan, W., \& Smith, J. D. (2009). Antibullying Programs in Schools: How Effective Are Evaluation Practices? Prevention Science, 10, 248-259.

Sallis, E., \& Jones, G. (2002). Knowledge Management in Education: Enhancing Learning \& Education. London: Kogan Page.

Senge, P. M. (2006). The Fifth Discipline. The Art \& Practice of the Learning Organization. London: Random House Business Books.

Sloane, P. (2006). The Leader's Guide to Lateral Thinking Skills: Unlocking the Creativity and Innovation in You and Your Team. London and Philadelphia, PA: Kogan Page Publishers.

Stephens, P. (2011). Preventing and Confronting School Bullying: A Comparative Study of Two National Programmes in Norway. British Educational Research Journal, 37, 381-404. http://dx.doi.org/10.1080/01411921003692868

Ttofi, M. M., \& Farrington, D. (2011). Effectiveness of School-Based Programs to Reduce Bullying: A Systematic and Meta-Analytic Review. Journal of Experimental Criminology, 7, 27-56.

Wickson, F., Carew, A. L., \& Russell, A. W. (2006). Transdisciplinary Research: Characteristics, Quandaries and Quality. Futures, 38, 1046-1059. http://dx.doi.org/10.1016/j.futures.2006.02.011 